\title{
Role perceptions of nurse clinical research coordinators
}

This article was published in the following Dove Press journal:

Nursing: Research and Reviews

24 September 2013

Number of times this article has been viewed

\section{Carolynn Thomas Jones Lynda L Wilson}

School of Nursing, University of Alabama at Birmingham, Birmingham, AL, USA
Correspondence: Carolynn Thomas Jones University of Alabama at Birmingham, School of Nursing, NB 4I4, I720 2nd Avenue South, Birmingham,

AL 35294-I210, USA

$\mathrm{Tel}+\mathrm{I} 2059343863$

Email cjthomas@uab.edu
Abstract: Nursing roles in clinical research have evolved in the last 3 decades and include diverse responsibilities and job titles. Nurse clinical research coordinators' (NCRCs) roles include study planning, implementation, participant recruitment and retention, assessment of participants' responses to clinical protocols, data management, and evaluation. The purpose of this study was to examine NCRCs' perceptions of 59 specific clinical research activities that have been proposed as a taxonomy of NCRC activities. Participants were asked to check whether each of the 59 activities is being performed, and whether those activities should be performed, by NCRCs. The sample included 61 NCRCs who were attending the annual meeting of the International Association of Clinical Research Nurses. The percentage of respondents who indicated that the 59 activities are being performed by NCRCs at their sites ranged from $55 \%-98.4 \%$. The percentage of respondents who indicated that the 59 activities should be performed by NCRCs ranged from $61.7 \%-88.5 \%$. There were eight activities that fewer than $70 \%$ of the respondents reported should be performed by NCRCs. Chi-square analyses were conducted to determine whether there was a difference in the distribution of responses to the "are performed" versus "should be performed" responses for each of the 59 activities. There were significant differences in the distributions for 49 of the activities. The percentage of nurses responding "are performed" was higher than the percentage of responses to the "should be performed" items for 41 of these 49 activities. Findings suggest that further research is needed to validate the extent to which the taxonomy of clinical research nurse (CRN) roles is a valid reflection of the actual practice of NCRCs, and also to explore reasons for the discrepancies in the responses to "are performed" and "should be performed" for 49 of these 59 activities that were identified in this study.

Keywords: clinical research nurse, study coordinator, research nurse coordinator, domains of practice in clinical research nursing

\section{Introduction}

Nursing roles in clinical research have evolved in the last 3 decades and include diverse responsibilities related to study planning, implementation, participant recruitment and retention, assessment of participants' responses to clinical protocols, data management, and evaluation. ${ }^{1-4}$ Mueller suggested that the role of the clinical research coordinator (CRC) has evolved as a result of investigators' delegation of clinical research roles and responsibilities to nurses. ${ }^{5}$ This transfer of the primary work of research coordination to nurses has led to the emergence of a new specialization in nursing. ${ }^{5,6}$ Because of competing responsibilities, physician investigators have transferred the majority of clinical research activities to coordinators and relied heavily on their study management, often resulting in the "PI" designation as meaning "practically invisible."” 
Job titles for nurses engaged in clinical research titles are diverse, and include titles such as study nurse, clinical research nurse, research nurse coordinator, clinical research coordinator, research nurse manager, research nurse practitioner, program manager, and clinical research manager. The Association of Clinical Research Professionals (ACRP) defines a CRC as an individual who, "regardless of job title, works at a clinical research site under the immediate direction of a principal investigator, whose research activities are conducted under Good Clinical Practice (GCP) guidelines" (p. 11). ${ }^{8}$ For the purpose of this paper, a nurse clinical research coordinator (NCRC) is a CRC who is also a nurse.

Study coordinators usually function with a high level of autonomy; however, they have reported both a lack of role clarity and isolation in their jobs. ${ }^{9}$ Several studies in the last decade have been conducted to define nursing competencies and activities of clinical research nurses and/ or NCRCs. Ehrenberger and Lillington developed a survey tool that included 154 items to identify the frequency and importance of clinical research nursing activities. ${ }^{10}$ The survey tool was finalized through focus group meetings with 24 geographically diverse clinical research nurses and ultimately validated in a survey by 40 oncology clinical research nurses. ${ }^{10}$

The National Institutes of Health Clinical Center formed an initiative called NIH $\mathrm{CRN}^{2010}$ to define the domains and develop a taxonomy of activities describing clinical research nursing. ${ }^{11}$ The taxonomy included 52 specific research activities across five domains of practice: clinical practice; care coordination and continuity; human subjects protections; study management; and contributing to the science. ${ }^{11}$ Castro et $\mathrm{al}^{12}$ conducted a Delphi survey with a 22 member expert panel. The findings from this study validated the five domains of practice and the 52 CRN activities defined by NIH CRN ${ }^{2010} .{ }^{11,12}$ Bevans et al expanded the list of CRN activities defined by Castro et al from 52 to 59 activities, splitting the items that contained more than one activity (Figure 1), and conducted a web-based survey to validate the revised taxonomy. ${ }^{13}$ The study sample included 412 nurses working in clinical research in two specific role areas at the NIH Clinical Center. The majority of respondents $(n=287 ; 69.7 \%)$ were clinical research nurses (CRN), defined as clinic nurse or staff nurse who implemented clinical research protocol orders as part of his/her staff nursing roles. A total of 74 were research nurse coordinators (RNCs), defined as nurses who were hired by principal investigators or departments at the NIH Clinical Center

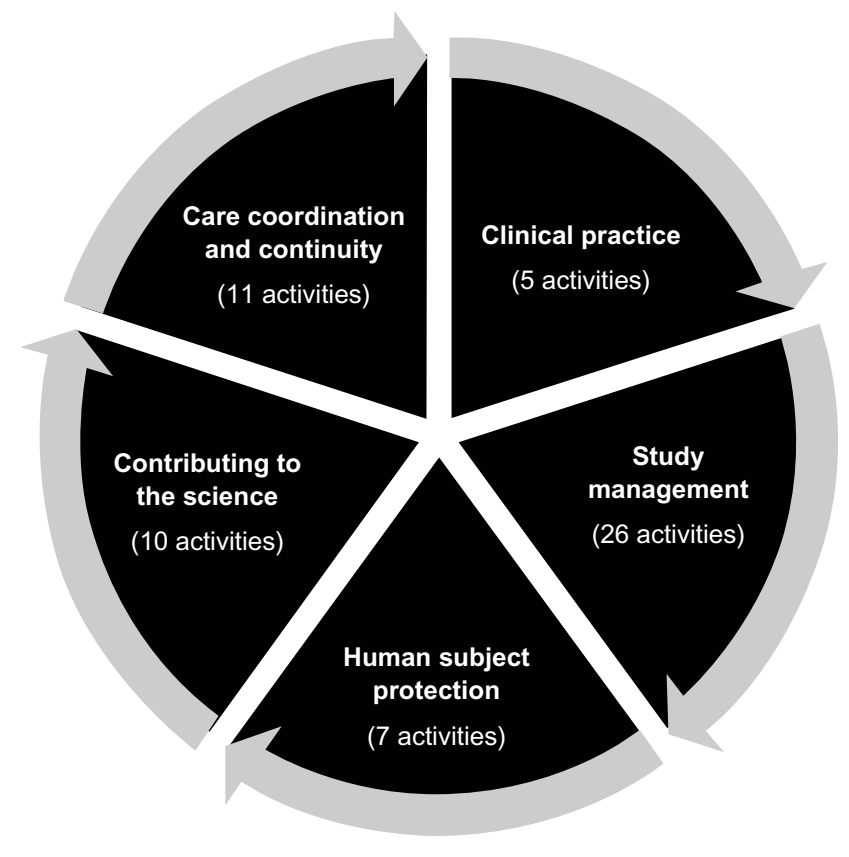

Figure I Domain of practice of clinical research nurses and number of activities.

to manage all aspects of one or more study protocols and were not working primarily as a staff nurse. ${ }^{13}$ The remaining participants were nurse practitioners or held other job titles $(\mathrm{n}=51 ; 12.4 \%)$. Respondents were asked to indicate the frequency with which they performed each of the activities in their research roles on a 6 -point Likert scale $(0=$ not part of my practice; 1 = infrequently, one to two times per year; $2=$ multiple times per year or monthly; $3=$ more than once per month; $4=$ once per day; and $5=$ multiple times per day). They were also asked to report the importance of each activity in their current role on a 6-point Likert scale $(0=$ not part of my role; $5=$ essential to my role). Findings indicated significant differences between the responses of the CRNs and RNCs for many activities, suggesting that these two roles are distinct. The CRNs reported significantly higher levels of activity frequency in the clinical practice domain and significantly lower levels of activity frequency in the study management, clinical care coordination, human subjects' protection, and contributing to science domains. There were few discrepancies between the responses to the reports of frequency and perceived importance of each of the 59 activities, suggesting that the respondents viewed most of the activities as important to the roles.

Although the findings indicated general support for the taxonomy, many respondents scored 0 or 1 for frequency for a number of the activities, indicating that they performed these activities less frequently than monthly. Because the current study focused on the roles of NCRCs, the findings 
related to the responses of the RNCs in the Bevans et al ${ }^{13}$ study were of particular interest. There were 20 activities that fewer than $70 \%$ of the CRCs reported performing at least monthly (range $0-68.5 \%$ ). These activities were in the study management $(n=12)$, human subjects protection $(n=2)$, and contributing to the science $(n=6)$ domains. The findings suggest the need for further research to determine whether other RNCs perceive that these activities are within the RNC scope of practice.

The purpose of the study reported in this paper was to further validate the taxonomy of clinical research activities that was published by Bevans et al, ${ }^{13}$ and to examine the perceptions of a different sample of NCRCs about whether these 59 activities are performed and should be performed by NCRCs. A secondary purpose was to identify activities that fewer than $70 \%$ of respondents perceive should be performed by NCRCs in order to identify activities in the taxonomy that may need to be revised. A third purpose was to determine whether there were differences in the distribution of responses to the "are performed" versus "should be performed" items for each of the 59 activities.

The specific study questions were:

1. For each of 59 specified clinical research activities, what percentage of respondents report that the activity is currently performed by NCRCs at their research sites?

2. For each of the 59 clinical research activities, what percentage of respondents report that the activity should be performed by NCRCs at their research sites?

3. Are there activities that fewer than $70 \%$ of respondents perceive should be performed by NCRCs at their research sites?

4. Is there a difference in the distribution of responses to the "is performed" versus "should be performed" items for each of the 59 activities?

\section{Participants and methods}

The University of Alabama at Birmingham Institutional Review Board provided expedited approval of this study. Because of the voluntary nature of the survey, and the lack of personal identifiers collected on the survey document, return of completed surveys indicated participants' consent to participate in the study.

\section{Participants}

The International Association of Clinical Research Nurses (IACRN) provided permission for the researcher to conduct the study during the 3rd IACRN Annual Meeting held in November 2011 in Bethesda, Maryland. A total of
229 nurses were registered to attend the meeting. In order to be eligible for the survey, respondents were required to be nurses who work in clinical research roles. During each day of the 3-day meeting, the researcher sat near the registration table to present the cover letter invitation and survey to participants. The participants were advised to complete only one survey and to place completed surveys in the labeled box on the registration table on any of the days during the meeting. The researcher also made periodic announcements during the meeting to remind participants to complete the survey.

\section{Data collection instrument}

Permission was obtained to utilize the 59-item clinical research nurse taxonomy instrument used in the Bevans et al study. ${ }^{13}$ Respondents were asked to respond to two questions for each of the 59 activities. Question A asked: "Who performs this activity at your site?" Question B asked: "Who should perform this activity at your site?" Selections for each question included three options: (1) nurse clinical research coordinators; (2) non-nurse clinical research coordinators with supervision; and (3) non-nurse clinical research coordinators without supervision. Respondents were advised to check all responses that applied, and thus for a given activity they may have checked all three options for each question. For the purpose of this paper, data for Questions A and B are being reported for role activity performance by nurse clinical research coordinators. Data for non-nurse clinical research coordinators are being reported elsewhere. ${ }^{14}$

\section{Results}

A total of 61 surveys (27\%) were returned. Data were analyzed using SPSS (version 21.0; IBM Corporation, Armonk, NY, USA) and Microsoft Excel for Mac 2011, Version 14.1.0 (Microsoft Corporation, Redmond, WA, USA). The highest level of education reported by respondents was associate degree/diploma (reported by 5 [8.5\%] of the respondents); Bachelor of Science/Bachelor of Arts (reported by 25 [42.4\%] of the respondents); Master of Arts/Master of Science (reported by 24 [40.7\%] of the respondents); and Doctorate (reported by 5 [8.5\%] of the respondents). The number of years of clinical research experience ranged from 1-39 years $($ mean $=13.48$ years; standard deviation $[\mathrm{SD}]=8.7$ ). The Cronbach's alpha internal consistency reliability of the list of activities was 0.951 for the responses to the "are performed" activities and 0.986 for the "should be performed" activities. 


\section{Findings related to study questions I, 2, and 3}

The percentage of respondents who indicated that the 59 activities are performed by $\mathrm{NCRCs}$ ranged from $55 \%-98.4 \%$, and the percentage of respondents who indicated that the 59 activities should be performed by NCRCs ranged from $61.7 \%-88.5 \%$ (Table 1 ). There were eight activities that fewer than $70 \%$ of the respondents reported should be performed by NCRCs; these are indicated with a superscript ${ }^{\mathrm{L}}$ in Table 1. Seven of these activities were in the study management domain, and one was in the contributing to the science domain.

\section{Findings related to study question 4}

A chi-square test for independence was performed with a Yates' continuity correction to determine whether there was a difference in the distribution of responses to the "are performed" versus "should be performed" items for each of the 59 activities. $P$-values for significant differences are illustrated in Table 1, revealing that there were 49 of the 59 activities with $P \leq 0.05$. The percentage of nurses who responded "are performed" was higher than the percentage of nurses that responded "should be performed" for 41 of these 49 activities. These findings may suggest that the respondents are experiencing a degree of conflict in their research roles, since there were significant differences in the distribution of "yes" responses to the "are performed" and "should be performed" categories for 49 of the 59 activities.

\section{Discussion}

Data from this study illustrate the wide range of activities that are being performed by NCRCs in clinical research sites, although the variation in responses to both the "are performed" and "should be performed" categories suggests that further work is needed to determine whether the Bevans et $\mathrm{al}^{13}$ taxonomy is a valid taxonomy for the role of the NCRC. Similar to the findings reported by Bevans et al, ${ }^{13}$ there were higher percentages of activities being performed in the clinical practice domain compared to the other domains. There were eight activities that fewer than $70 \%$ of respondents indicated should be performed by NCRCs. Four of these activities were in the list of 20 activities that fewer than $70 \%$ of the RNCs in the Bevans et al ${ }^{13}$ study reported to have performed at least monthly. These items were all in the study management domain and included: support for study budget development; support study grant development; participate in the identification of research trends; and participate in the setup of study-specific databases. The findings from this study cannot be directly compared to those reported by Bevans et $\mathrm{al}^{13}$ because the questions asked in the two studies were different. Bevans et $\mathrm{al}^{13}$ asked respondents to indicate the frequency with which they performed each of the 59 activities, using a 6-point Likert scale $(0=$ not part of my role; $5=$ multiple times per day). In the current study, the respondents were simply asked to indicate whether each activity is performed and should be performed by NCRCs. Despite these differences, it may be useful to examine whether the four items with low frequencies in both studies are indeed appropriate components of the NCRC role.

The finding that there were significant differences in the distribution of responses to the "are performed" and "should be performed" questions for 49 of the 59 activities suggests that the respondents may be experiencing some degree of role conflict. The finding that the percentage of nurses responding "are performed" was higher than the percentage of responses to the "should be performed" for 41 of these 49 activities suggests that respondents may not be certain that these activities are appropriate for the NCRC. Multiple factors could influence NCRC perceptions of their role activities. Additional research is needed to validate the Bevans et $\mathrm{al}^{13}$ taxonomy and to clarify the role of the NCRC.

\section{Limitations}

Although this study provided important information to examine the taxonomy of NCRC roles, there were several limitations of this study. The small convenience sample was recruited at the IACRN annual meeting and included a large number of clinical research nurses employed at the US National Institutes of Health (NIH) and at academic medical centers. These participants may not be representative of all clinical research nurses in the US or globally. Because materials were not included in the meeting packets, there was no guarantee that all potential participants were given a survey; therefore, estimates for participation may be under-reported. Furthermore, not all attendees were present during the full 3-day conference and may have missed reminders to turn in the survey. Moreover, data were not collected to describe the types of research being performed by attendees by disease or acuity. Perceptions of nurse activities may have been influenced by such contexts. The use of a Likert scale may have strengthened data collection on perceptions of whether nurses should perform the specific activities. An evaluation of clinical research efficacy would likewise strengthen the interpretation of results. Finally, collecting data about the specific research roles of the respondents would have allowed for a comparison of responses based on 
Table I Percentage of respondents reporting that NCRCs "do" and "should" perform activities: analyzed by experience and education

\begin{tabular}{|c|c|c|c|}
\hline Clinical research activity by domain of practice & $\begin{array}{l}\text { Do perform } \\
\text { (\%) }\end{array}$ & $\begin{array}{l}\text { Should perform } \\
\text { (\%) }\end{array}$ & $\frac{\text { Chi-square }^{\dagger}}{P \text {-value }}$ \\
\hline \multicolumn{4}{|l|}{ DOMAIN: care coordination and continuity $(\mathrm{CC})$} \\
\hline $\begin{array}{l}\text { Collaborate with the interdisciplinary team to create and communicate a plan of care } \\
\text { that allows for safe and effective collection of clinical research data (CC) }\end{array}$ & 96.7 & 77 & NS** \\
\hline Provide nursing leadership within the interdisciplinary team (CC) & 93.4 & 80.3 & 0.000 \\
\hline Communicate the impact of study procedures on the research participants (CC) & 98.4 & 85.2 & NS** \\
\hline $\begin{array}{l}\text { Provide nursing expertise to community based health care personnel (eg, referring } \\
\text { physician or center) related to study participation (CC) }\end{array}$ & 85.2 & 77 & 0.003 \\
\hline Coordinate interdisciplinary meetings and activities in the context of a study (CC) & 93.4 & 80.3 & 0.026 \\
\hline $\begin{array}{l}\text { Coordinate referrals to appropriate interdisciplinary services outside the immediate } \\
\text { research team }(C C)\end{array}$ & 85.2 & 75.4 & 0.000 \\
\hline Coordinate research participant study visits (CC) & 86.9 & 72.1 & 0.000 \\
\hline Provide indirect nursing care in the context of research participation (CC) & 90.2 & 77 & 0.000 \\
\hline Facilitate research participant inquiries and concerns $(\mathrm{CC})$ & 88.5 & 80.3 & 0.000 \\
\hline Facilitate the education of the interdisciplinary team on study requirements (CC) & 91.8 & 82 & 0.002 \\
\hline \multicolumn{4}{|l|}{ DOMAIN: clinical practice (CP) } \\
\hline Provide direct nursing care to research participants (CP) & 96.7 & 78.7 & NS** \\
\hline Monitor the research participant for potential adverse events $(\mathrm{CP})$ & 98.4 & 85.2 & NS** \\
\hline Report potential adverse events to a member of the research team (CP) & 98.4 & 78.7 & NS** \\
\hline Record research data in approved source document $(\mathrm{CP})$ & 90.2 & 77 & 0.001 \\
\hline $\begin{array}{l}\text { Provide teaching to research participants and family regarding study participation, } \\
\text { participant's current clinical condition, and/or disease process (CP) }\end{array}$ & 95.1 & 86.9 & 0.000 \\
\hline \multicolumn{4}{|l|}{ DOMAIN: contributing to the science (CS) } \\
\hline Perform secondary data analysis to contribute to the development of new ideas (CS) & 55.7 & $63.9 *, \mathrm{~L}$ & 0.002 \\
\hline $\begin{array}{l}\text { Identify questions appropriate for clinical nursing research as a result of study team } \\
\text { participation (CS) }\end{array}$ & 82 & 77 & $0.002 * *$ \\
\hline $\begin{array}{l}\text { Collaborate with the interdisciplinary team to develop innovations in care delivery that } \\
\text { have the potential to improve patient outcomes and accuracy of data collection (CS) }\end{array}$ & 96.7 & 80.3 & $0.048 * *$ \\
\hline Serve as a resource to new investigators (CS) & 95.1 & 80.3 & $0.004 * *$ \\
\hline $\begin{array}{l}\text { Serve as an expert in a specialty area (eg, grant reviewer, editorial board, } \\
\text { presenter, etc) (CS) }\end{array}$ & 68.9 & $70.5^{*}$ & 0.000 \\
\hline Participate in the query of research data to prepare for analysis (CS) & 73.3 & $73.8^{*}$ & $0.000 * *$ \\
\hline Participate in the analysis of research data (CS) & 57.4 & $72.1 *$ & 0.000 \\
\hline Generate practice questions as a result of a new study procedure or intervention (CS) & 82 & $82 *$ & $0.000 * *$ \\
\hline $\begin{array}{l}\text { Disseminate clinical expertise and best practices related to clinical research through } \\
\text { presentations, publications, and/or interactions with nursing colleagues (CS) }\end{array}$ & 90.2 & 88.5 & $0.015 * *$ \\
\hline Mentor junior staff and students participating as members of the research team (CS) & 85.2 & 82 & $0.007 * *$ \\
\hline \multicolumn{4}{|l|}{ DOMAIN: human subject protection (HSP) } \\
\hline Serve as IRB member (HSP) & 57.4 & $73.8 *$ & 0.001 \\
\hline Collaborate with the interdisciplinary team to address ethical conflicts (HSP) & 95.1 & 82 & NS** \\
\hline Facilitate the initial informed consent/assent process (HSP) & 90.2 & 78.7 & NS** \\
\hline Manage potential ethical and financial conflicts of interest for self (HSP) & 83.6 & 75.4 & $0.000 * *$ \\
\hline $\begin{array}{l}\text { Support research participant in defining his/her reasons and goals for participating } \\
\text { in a study (HSP) }\end{array}$ & 85.2 & 72.1 & $0.000 * *$ \\
\hline Coordinate research activities to minimize subject risk (HSP) & 90.2 & 78.7 & $0.000 * *$ \\
\hline Facilitate the ongoing informed consent/assent process (HSP) & 91.8 & 83.6 & $0.000 * *$ \\
\hline \multicolumn{4}{|l|}{ DOMAIN: study management (SM) } \\
\hline Participate in research participant recruitment (SM) & 80.3 & 72.1 & $0.000 * *$ \\
\hline Facilitate scheduling of study procedures (SM) & 85.2 & $62.3^{\mathrm{L}}$ & $0.002 * *$ \\
\hline Support study budget development (SM) & 66.7 & $61.7^{2}$ & 0.000 \\
\hline Collect data on research participant based on study endpoints (SM) & 82 & 77 & $0.000 * *$ \\
\hline Contribute to the development of case report forms (SM) & 75.4 & $75.4^{*}$ & $0.000 * *$ \\
\hline Coordinate the collection of research specimens (SM) & 95.1 & 80.3 & NS** \\
\hline Facilitate processing of research specimens (SM) & 85 & $66.7^{\mathrm{L}}$ & $0.000 * *$ \\
\hline Provide nursing expertise to the research team during study development (SM) & 90.2 & 82 & $0.007 * *$ \\
\hline Record data on official study documents (SM) & 86.9 & 75.4 & $0.000 * *$ \\
\hline
\end{tabular}


Table I (Continued)

\begin{tabular}{|c|c|c|c|}
\hline Clinical research activity by domain of practice & $\begin{array}{l}\text { Do perform } \\
\text { (\%) }\end{array}$ & $\begin{array}{l}\text { Should perform } \\
\text { (\%) }\end{array}$ & $\frac{\text { Chi-square }^{\dagger}}{P \text {-value }}$ \\
\hline Participate in the reporting of research trends (SM) & 75.4 & 73.8 & $0.016 * *$ \\
\hline Comply with International Conference on Harmonization (ICH) Good Clinical & 90.2 & 78.7 & $0.00 I^{* *}$ \\
\hline \multicolumn{4}{|l|}{ Practice guidelines (SM) } \\
\hline Perform quality assurance activities to assure data integrity (SM) & 83.6 & 77 & $0.001 * *$ \\
\hline Facilitate accurate communication among research sites (for multisite studies) (SM) & 83.6 & 70.5 & $0.000 * *$ \\
\hline Participate in screening potential research participants for eligibility (SM) & 85.2 & 73.8 & $0.000 * *$ \\
\hline Support study grant development (SM) & 55 & $65^{*, L}$ & 0.000 \\
\hline $\begin{array}{l}\text { Identify clinical care implications during study development (eg, staff competencies } \\
\text { and resources, equipment, etc) (SM) }\end{array}$ & 86.9 & 85.2 & NS* \\
\hline $\begin{array}{l}\text { Participate in the preparation of reports for appropriate regulatory and monitoring } \\
\text { bodies/boards (SM) }\end{array}$ & 80.3 & 75.4 & $0.000 *$ \\
\hline Participate in the identification of research trends (SM) & 63.9 & $68.9^{*, \mathrm{~L}}$ & 0.000 \\
\hline Participate in study development (SM) & 72.1 & $75.4^{*}$ & $0.000 *$ \\
\hline Develop study specific materials for research participant education (SM) & 90.2 & 85.2 & $0.002 *$ \\
\hline Participate in site visits and/or audits (SM) & 95.1 & 83.6 & $0.00 I^{*}$ \\
\hline Oversee human resources (people) related to research process (SM) & 90 & 85 & $0.002 *$ \\
\hline Protect research participant data in accordance with regulatory requirements (SM) & 93.4 & 85.2 & $0.005 *$ \\
\hline Participate in the set-up of a study specific database (SM) & 68.9 & $63.9^{\mathrm{L}}$ & 0.000 \\
\hline Facilitate communication within the research team (SM) & 95.1 & 83.3 & NS* \\
\hline Facilitate the handling (storage and shipping) of research specimens (SM) & 73.8 & $62.3^{\mathrm{L}}$ & $0.000^{*}$ \\
\hline Provide nursing expertise to the research team during study implementation (SM) & 95.1 & 85.2 & $0.000 *$ \\
\hline
\end{tabular}

Notes: *Indicates where "should perform" percentage was the same or higher than the "do perform" percentage; 'indicates that the percentage of respondents reporting "Should perform" was lower than 70\%; tindicates chi-square for independence, $X^{2}(\mathrm{I}, \mathrm{n}=6 \mathrm{I})$ with Yates' continuity correction; **further describes Yates continuity correction for items $<5$, per chi-square analyses in SPSS.

Abbreviations: NCRCs, nurse clinical research coordinators; IRB, institutional review board; NS, not significant.

different research roles, similar to the analysis conducted by Bevans et al. ${ }^{13}$

\section{Conclusion}

Despite limitations, the findings from this study provide further assessment of the extent to which the taxonomy proposed by Bevans et al is a valid reflection of the NCRC role. ${ }^{13}$ The study provided additional information by comparing nurses' perceptions of activities that are performed by NCRCs compared to activities that should be performed by these nurses. These findings suggest the need for further discussion and research to clarify the activities and competencies required for this important nursing role. Such clarification will be essential in guiding development of educational programs to ensure that nurses are prepared to fulfill this important role.

\section{Acknowledgments}

The authors would like to thank Clare Hastings, RN, PhD, FAAN, NIH Clinical Center, for her valuable comments in preparing this survey study, and Jennifer Cunningham, UAB $\mathrm{PhD}$ Student in Health Education, for her assistance with data entry and quality checks.

\section{Author contributions}

All authors made substantial contributions to this study in terms of: (1) the conception and design, acquisition of data, or analysis and interpretation of data; (2) drafting the article or reviewing it critically for important intellectual content; and (3) giving final approval of the version to be published.

\section{Disclosure}

The authors report no conflicts of interest in this work.

\section{References}

1. Raybuck J. The clinical nurse specialist as research coordinator in clinical drug trials. Clin Nurse Spec. 1997;11:15-19.

2. Bell J. Towards clarification of the role of research nurses in New Zealand: a literature review. Nurs Prax N Z. 2009;25(1):4-16.

3. Hastings C, Fisher CA, McCabe MA, et al. Clinical research nursing: A critical resource in the national research enterprise. Nurs Outlook. 2012;60(3):149-156.

4. Mori C, Mullen N, Hill EE. Describing the role of the clinical research nurse. Research Practitioner. 2007;8(6):220-228.

5. Mueller M. From delegation to specialization: nurses and clinical trial co-ordination. Nur Inq. 2001;8(3):182-190.

6. Baer A, Zon R, Devine S, Lyss A. The clinical research team. J Oncol Pract. 2011;7(3):188-192.

7. Schramp LC, Holtcamp M, Phillips SA, Johnson TP, Hoff J. Advance practice nurses facilitating clinical translational research. Clin Med Res. 2010;8(3-4):131-134. 
8. Association of Clinical Research Professionals. CRC Certification Guide. Alexandria, VA: Association of Clinical Research Professionals; 2011. Available from: http://www.acrpnet.org/PDF/CRC_Guide.pdf. Accessed June 2, 2013.

9. Raja-Jones H. Role boundaries - research nurse or clinical nurse specialist? A literature review. J Clin Nurs. 2002;11(4):415-420.

10. Ehrenberger HE, Lillington L. Development of a measure to delineate the clinical trials nursing role. Oncol Nurs Forum. 2004;31(3): E64-E68.

11. Clinical Research Nurse 2010 Domain of Practice Committee. Building The Foundation for Clinical Research Nursing: Domain of Practice for the Specialty of Clinical Research Nursing. Bethesda: National Institutes of Health (NIH); 2009. Available from: http://www.cc.nih. gov/nursing/crn/DOP_document.pdf. Accessed April 13, 2013.
12. Castro K, Bevans M, Miller-Davis C, et al. Validating the clinical research nursing domain of practice. Oncol Nurs Forum. 2011;38(2): E72-E80.

13. Bevans M, Hastings C, Wehrlen L, et al. Defining clinical research nurse practice: results of a role delineation study. Clin Transl Sci. 2011;4(6):421-427.

14. Jones CT, Wilson LL. Clinical research nurses' perceptions of research activities performed by unsupervised non-nurse clinical research coordinators. The Monitor. Epub 2013 July.

Nursing: Research and Reviews

\section{Publish your work in this journal}

Nursing: Research and Reviews is an international, peer-reviewed, open access journal publishing original research, reports, reviews and commentaries on all aspects of nursing and patient care. These include patient education and counselling, ethics, management and organizational issues, diagnostics and prescribing, economics and

\section{Dovepress}

resource management, health outcomes, and improving patient safety in all settings. The manuscript management system is completely online and includes a very quick and fair peer-review system. Visit http://www.dovepress.com/testimonials.php to read real quotes from published authors.

Submit your manuscript here: http://www.dovepress.com/nursing-research-and-reviews-journal 